\title{
The Use of Project Time Management Processes and the Schedule Performance of Construction Projects in Mexico
}

\author{
Rómel G. Solís-Carcaño, Gilberto A. Corona-Suárez, and Aldo J. García-Ibarra \\ Faculty of Engineering, Autonomous University of Yucatan, Avenida Industrias No Contaminantes, 97203 Merida, YUC, Mexico \\ Correspondence should be addressed to Gilberto A. Corona-Suárez; csuarez@uady.mx
}

Received 30 June 2015; Accepted 17 September 2015

Academic Editor: Eric Lui

Copyright @ 2015 Rómel G. Solís-Carcaño et al. This is an open access article distributed under the Creative Commons Attribution License, which permits unrestricted use, distribution, and reproduction in any medium, provided the original work is properly cited.

\begin{abstract}
Delays have been frequently reported as the cause of several conflicts that affect the different parties involved in construction projects. Project Time Management (PTM) includes a number of planning and controlling processes that are recommended for complying with requirements related to project time. The study reported in this paper aimed at assessing the use of PTM processes and its relation with project schedule performance (i.e., timely completion). Seven PTM processes and seventy-seven tasks associated with them were identified from the literature that is globally relevant to project management. The study included the assessment of fourteen school construction projects executed by a public agency in the Yucatan Peninsula, Mexico. These projects were monitored during the construction phase in order to measure two different variables: the use of processes related to PTM (i.e., schedule planning and controlling processes) and the project schedule performance. For each of these projects a Use Index was obtained for assessing the first variable, while the Schedule Performance Index and the Schedule Variance were computed to assess the second one. The results demonstrated there is statistical dependence between these two variables. Most of the projects that attained timely completion also made a greater use of the PTM processes.
\end{abstract}

\section{Introduction}

The delays on the delivery of construction projects are seen as one of the most frequent problems in the construction industry [1]. In a previous study, in the same specific location where the present study was carried out, $28 \%$ of the contractors participating in this study pondered construction delays were very frequent, and $61 \%$ regarded delays as fairly frequent, while only $11 \%$ indicated that they were infrequent [2]. Thus, in the context studied in this case, it seems quite common to have contractors failing to complete construction projects on time.

The aftermath of delays affects all people and organizations involved in the project. This is especially true for the owner's business since delaying the startup of the project will impede obtaining the expected project revenue and will increase financial costs. In addition, the owner may face several other difficulties resulting from the commitments assumed based on the delivery date established in the contract [3]. On the other hand, prolonging the project execution time usually results in contractors that have to deal with cost overruns due mostly to the following causes: extra expenses on management personnel, cost escalations of materials, increase of financial cost, paying contract penalties, and so forth [4]. Moreover, given the usual competitive environment in the construction industry, contractors that fail to complete projects on time may get their reputation harmed and become impeded to obtain new contracts.

Project quality can also be affected due to construction delays since the construction team usually dedicates less time to quality control when the main concern is completing the project on time. When this is the case, workers are usually pushed to work overtime and to increase the production rate, which very often entails failures and reworks [5].

In developing countries delays during the construction of public assets, such as schools, could also result in social harm given the fact that this kind of infrastructure is usually urgently needed. Therefore, the sooner those projects are completed, the better for satisfying the social needs in those countries.

In order to avoid the aforementioned incidents, contractors should implement project management processes 
that lead to success in the delivery of construction projects. Project management includes a number of planning and controlling processes that should be applied to comply with the owners' requirements related to project time, cost, and quality [6]. In fact, project management has evolved towards a sophisticate and comprehensive process that depicts the primary approach to succeed on the delivery of any project. Since knowledge on project management has been developing over time, several professional associations around the world have issued guidelines and standards to put into practice such process. For instance, the Project Management Institute (PMI) has issued five editions of the PMBOK (Project Management Body of Knowledge) Guide [7], while the Association for Project Management (APM) has so far released the sixth edition of the APM Body of Knowledge [8]. The standardization of the project management process contributes to disseminate best practices which implementation depicts a strategy to improve project performance [9].

Regarding the prevention of project delays, the PMBOK Guide dedicates one of ten Knowledge Areas to Project Time Management, which includes the processes required to accomplish timely completion of the project [7]. This knowledge area includes processes such as Activity Definition, Activity Sequencing, Activity Resource Estimating, Activity Duration Estimating, Schedule Development, and Schedule Control. The appropriateness of Project Time Management can be seen as a relevant indicator that could be used to assess contractors' effectiveness and capability to succeed on the completion of a project and to evaluate contractors' performance [10].

The study reported in this paper aimed at assessing the use of processes related to Project Time Management and its relation with project performance regarding timely completion.

\section{Research Methods}

The study included the assessment of fourteen school construction projects executed by different construction firms serving the same client: a public agency dedicated to the development of school infrastructure in the Yucatan Peninsula, Mexico. These projects were monitored during the construction phase in order to measure two different variables: the degree of use of processes related to Project Time Management (PTM) and the project performance level regarding timely completion. In order to restrain the effect of key factors that may influence project performance, the following extraneous variables were fixed: the client (all contractors in charge of the projects were contracted by the same government agency); the type of construction project (all of them were school buildings); the design (similar architecture and materials for all projects); the construction time period (all projects were constructed simultaneously); the environmental context (all projects were executed in the Yucatan Peninsula, Mexico); and the specialty of contractors (all projects executed by construction firms mostly dedicated to the same building type).

2.1. Measuring the Use of PTM Processes. The practices, tools, and techniques that the PMI recommends were considered in order to assess the extent to which the PTM processes were used in the execution of the projects included in this study. The PMBOK Guide [7] was used to identify the PTM processes that need to be in place to complete construction projects on time. According to the purpose of the PTM processes they can be related to the planning (i.e., Activity Definition, Activity Sequencing, Activity Resource Estimating, Activity Duration Estimating, and Schedule Development) or to controlling (i.e., Schedule Monitoring and Schedule Control) of the project. In addition, more specific tasks, associated with each of the identified processes, were recognized within the descriptions given for these processes. A total of seven processes and seventy-seven tasks were identified. Table 1 shows some examples of the tasks associated with some of the PTM processes. In addition, Table 2 shows the number of tasks associated with each PTM process.

An instrument was designed to assess the use of the identified PTM processes and tasks. This instrument was applied to personnel involved in the planning and controlling of any of the fourteen projects observed in this study. The respondents had to mark, from the tasks listed in the instrument, those that had been used for the planning and controlling of the projects in which they were involved. An index depicting the extent to which a PTM process was used was then computed as the ratio of the number of tasks marked by the respondent and the total of tasks associated with that process. A Use Index (UI) depicting the extent to which a PTM process was used was then computed as the ratio of the number of tasks marked by the respondent and the total of tasks associated with that process. An UI was calculated for the planning function as the ratio of the number of tasks associated with the planning processes that were marked by the respondent and the total of tasks associated with all of such processes. The same approach was used to calculate a UI for the controlling function as well as for a global one (i.e., considering all the tasks listed in the instrument).

2.2. Measuring the Schedule Performance. The schedule performance of the projects observed in this study was assessed every week during the execution phase. This assessment was conducted according to the Earned Schedule (ES) approach introduced by Lipke [11], which includes the following.

(a) ES (Earned Schedule) is the point in time at which the cumulative amount of Earned Value (EV) should have been attained in accordance with the schedule [11]. According to the PMBOK Guide [7], the project EV can be obtained as the Budgeted Cost of Work Performed (BCWP) of the project. Therefore, as shown in Figure 1, ES can be estimated by projecting the cumulative amount of EV against the curve depicting the Budgeted Cost of Work Scheduled (BCWS). The vertical line from the point intersected on the BCWS curve to the horizontal axis (time) determines the earned portion of the schedule. The duration from the beginning of the project to the intersection of the time axis is the ES. 
TABLE 1: Examples of PTM processes and corresponding tasks [7].

\begin{tabular}{ll}
\hline Processes & Associated tasks \\
\hline & To identify project work packages and decompose into schedule activities to provide a basis for estimating, \\
scheduling, executing, and monitoring and controlling the project work. \\
To consider enterprise environmental factors and organizational process assets, as well as the project deliverables, \\
constraints, and assumptions documented in the project scope statement. \\
To elaborate an activity list that includes all schedule activities that are planned to be performed on the project (i.e., \\
it does not include any schedule activities that are not required as part of the project scope). \\
To elaborate the scope of work description for each schedule activity in sufficient detail to ensure that project team \\
members understand what work is required to be completed. \\
To determine the attributes associated with each schedule activity (e.g., the activity identifier, activity codes, \\
activity description, predecessor and successor activities, leads and lags, and resource requirements). \\
To obtain the list of schedule milestones identifying all milestones and indicating whether the milestone is \\
mandatory or optional. \\
To generate requested changes that can affect the project scope statement and WBS.
\end{tabular}

To consider historical information maintained as part of organizational process assets, as well as the constraints and assumptions from the project scope statement.

To consider the estimated activity resource requirements and resource calendars since resources assigned to the Activity duration schedule activity, and the availability of such resources, will significantly influence the duration of most activities. estimating To estimate activity durations through techniques such as expert judgment, analogous estimating, parametric estimating, three-point estimates, or reserve analysis.

To update the activity attributes to include the durations for each schedule activity, the assumptions made in developing the activity duration estimates, and any contingency reserves.

To implement the schedule change control system that defines the procedures by which the project schedule can be changed. It includes the paperwork, tracking systems, and approval levels necessary for authorizing changes.

To measure the progress of schedule activities in order to obtain the data required for determining the project schedule performance.

To report the progress and current schedule status, including information such as actual start and finish dates, remaining durations for unfinished schedule activities, and the percent complete of in-progress schedule activities. To use a comparison bar displaying two bars: one depicting the current actual status and the other the status of the Schedule monitoring approved project schedule baseline for each schedule activity, in order to facilitate analysis of schedule progress. To update the project schedule for determining if the schedule baseline has changed; this includes developing new project schedule network diagrams to display approved remaining durations and modifications to the work plan.

To develop, when project schedule delays are severe, a new target schedule with revised target start and finish dates, in order to provide realistic data for directing the work, and for measuring performance and progress.

To calculate schedule variance (SV) and schedule performance index (SPI) values for WBS components, in order to provide useful information for the detection of deviations.

TABLE 2: Number of tasks associated with each PTM process.

\begin{tabular}{lcc}
\hline Function & Processes & $\begin{array}{c}\text { Number of } \\
\text { associated tasks }\end{array}$ \\
\hline \multirow{3}{*}{ Schedule } & Activity definition & 7 \\
planning & Activity sequencing & 12 \\
& Activity resource estimating & 7 \\
& Activity duration estimating & 4 \\
& Schedule development & 23 \\
\hline Schedule & Schedule monitoring & 7 \\
controlling & Schedule control & 17 \\
\hline
\end{tabular}

(b) AT (Actual Time) is the actual time duration from the initiation of the project to the point in time at which the schedule performance of the project is assessed. (c) The Schedule Performance Index (SPI) depicts time performance of construction work as the quantitative relation between the units of time matching the point ES and the units of time corresponding to the AT, as indicated in

$$
\mathrm{SPI}(t)=\frac{\mathrm{ES}}{\mathrm{AT}}
$$

(d) The Schedule Variance (SV) assesses time performance in absolute terms by subtracting the units of time corresponding to the AT to the units of time matching the ES, as indicated in

$$
\mathrm{SV}(t)=\mathrm{ES}-\mathrm{AT} \text {. }
$$

Both, the SPI and the SV, are indicators that can be used for decision making regarding the need to implement actions to overcome construction delays. 
TABLE 3: Information of contractors and projects observed in this study.

\begin{tabular}{|c|c|c|c|c|}
\hline \multirow[b]{2}{*}{ ID } & \multicolumn{2}{|c|}{ Information of contractors } & \multicolumn{2}{|c|}{ Project } \\
\hline & Business size & $\begin{array}{l}\text { Portion of annual production dedicated to school } \\
\text { construction projects }\end{array}$ & $\begin{array}{l}\text { Total budget } \\
\text { (US dollars) }\end{array}$ & $\begin{array}{c}\text { Scheduled } \\
\text { duration (days) }\end{array}$ \\
\hline 1 & Small & $41-60 \%$ & $\$ 219,673$ & 91 \\
\hline 2 & Micro & $81-95 \%$ & $\$ 228,569$ & 84 \\
\hline 3 & Medium & $21-40 \%$ & $\$ 192,009$ & 91 \\
\hline 4 & Micro & $81-95 \%$ & $\$ 140,004$ & 77 \\
\hline 5 & Medium & $61-80 \%$ & $\$ 455,572$ & 98 \\
\hline 6 & Medium & $61-80 \%$ & $\$ 242,209$ & 112 \\
\hline 7 & Small & $81-95 \%$ & $\$ 177,019$ & 98 \\
\hline 8 & Small & $21-40 \%$ & $\$ 182,319$ & 97 \\
\hline 9 & Micro & $41-60 \%$ & $\$ 130,739$ & 98 \\
\hline 10 & Small & $41-60 \%$ & $\$ 121,811$ & 63 \\
\hline 11 & Small & $96-100 \%$ & $\$ 547,710$ & 105 \\
\hline 12 & Medium & $21-40 \%$ & $\$ 128,293$ & 84 \\
\hline 13 & Medium & $6-20 \%$ & $\$ 73,428$ & 98 \\
\hline 14 & Medium & $21-40 \%$ & $\$ 177,879$ & 98 \\
\hline
\end{tabular}

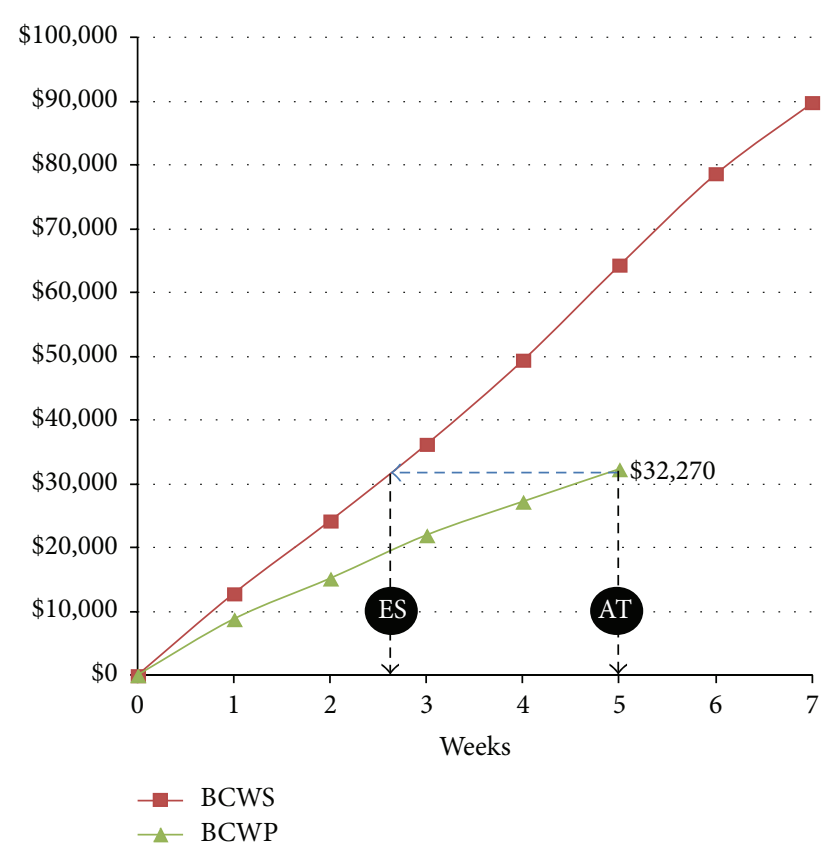

FIgURE 1: Example of the estimating of ES.

The estimating of ES is illustrated with the estimating conducted for one of the projects observed in this study. Figure 1 shows that, according to the BCWP curve, the project cumulative EV was $\$ 32,270$ (US dollar) at the end of week 5 (AT). By projecting the point defined by such time (week 5) and value $(\$ 32,270)$ on the BCWS curve, the ES resulted 2.78 weeks. This meant that by the end of week 5 the project work had an actual progress that should be completed by week 2.78 . Using these values on (1) and (2), SPI(5) resulted 0.56 (i.e., the proportion of work that was actually completed) and the outcome for SV(5) was -2.22 weeks (i.e., the amount of time the project schedule was behind the planned progress).
With the computing of the SPI and the SV, those projects that concluded within the time required by the client were identified, as well as those that did not. From the last ones, those projects for which the client granted an extension of time were identified as well.

2.3. Analyzing the Relation between UI and Schedule Performance. Since a single value of UI was assessed for each of the projects observed in this study, while several (weekly) values of SPI and SV could be estimated for each of them, schedule performance assessment for each project was based on the corresponding SPI and SV values estimated at four different periods of time: from the beginning to the end of the fourth, eighth, twelfth, and last weeks (i.e., this is variable for each project) of the project.

Correlation analysis was the statistical method used to determine the extent to which schedule performance (i.e., SPI and SV values) in each project was dependent on the use of PTM processes (i.e., UI values). Correlation coefficients $(r)$ were computed for each project at the aforesaid periods of time. Based on the obtained $r$ values, the criteria proposed by Wadsworth [12] were used to assess the strength of the statistical relationship between the studied variables, included the following:

(i) moderate relationship: from $r=0.40$ to 0.59 ,

(ii) strong relationship: from $r=0.60$ to 0.79 ,

(iii) very strong relationship: from $r=0.80$ to 1.00 .

\section{Results}

Table 3 informs the business size of the firms that participated in this study, as well as the portion of their annual production corresponding to school infrastructure construction. The criteria from INEGI [13] were used to classify these organizations according to their sizes, which is based on the number 
Table 4: Use Indexes (UI) for the Project Time Management processes.

\begin{tabular}{|c|c|c|c|c|c|c|c|c|c|c|c|c|c|c|}
\hline \multirow{2}{*}{ Processes } & \multicolumn{14}{|c|}{ Projects } \\
\hline & 1 & 2 & 3 & 4 & 5 & 6 & 7 & 8 & 9 & 10 & 11 & 12 & 13 & 14 \\
\hline UI for schedule planning & 0.51 & 0.53 & 0.40 & 0.51 & 0.60 & 0.60 & 0.60 & 0.60 & 0.55 & 0.38 & 0.51 & 0.40 & 0.53 & 0.38 \\
\hline Activity definition & 0.60 & 0.80 & 0.60 & 0.80 & 1.00 & 0.80 & 0.80 & 1.00 & 0.40 & 0.40 & 1.00 & 0.60 & 0.40 & 0.60 \\
\hline Activity sequencing & 0.67 & 0.67 & 0.50 & 0.58 & 0.83 & 0.67 & 0.67 & 0.75 & 0.58 & 0.33 & 0.58 & 0.33 & 0.58 & 0.42 \\
\hline Activity resource estimating & 0.57 & 0.43 & 0.29 & 0.71 & 0.29 & 0.29 & 0.43 & 0.57 & 0.57 & 0.57 & 0.14 & 0.57 & 0.71 & 0.57 \\
\hline Activity duration estimating & 0.80 & 0.40 & 0.40 & 0.40 & 0.80 & 0.40 & 0.40 & 0.60 & 0.80 & 0.40 & 0.60 & 0.40 & 0.20 & 0.20 \\
\hline Schedule development & 0.33 & 0.46 & 0.33 & 0.38 & 0.46 & 0.67 & 0.63 & 0.46 & 0.50 & 0.33 & 0.46 & 0.33 & 0.54 & 0.29 \\
\hline UI for schedule controlling & 0.33 & 0.46 & 0.25 & 0.33 & 0.46 & 0.42 & 0.38 & 0.75 & 0.33 & 0.38 & 0.54 & 0.21 & 0.33 & 0.42 \\
\hline Schedule monitoring & 0.35 & 0.47 & 0.29 & 0.35 & 0.53 & 0.41 & 0.41 & 0.71 & 0.41 & 0.47 & 0.47 & 0.24 & 0.35 & 0.47 \\
\hline Schedule control & 0.67 & 1.00 & 0.33 & 0.67 & 0.33 & 0.67 & 0.67 & 1.00 & 0.33 & 0.33 & 1.00 & 0.33 & 0.67 & 0.67 \\
\hline Total UI & 0.45 & 0.51 & 0.35 & 0.45 & 0.56 & 0.55 & 0.53 & 0.65 & 0.48 & 0.38 & 0.52 & 0.34 & 0.47 & 0.39 \\
\hline
\end{tabular}

TABLe 5: Results of the Schedule Performance Indexes in the observed projects.

\begin{tabular}{|c|c|c|c|c|c|c|c|c|c|c|c|c|c|c|}
\hline \multirow{2}{*}{ Weeks } & \multicolumn{7}{|c|}{ Delayed projects } & \multicolumn{7}{|c|}{ Projects completed on time } \\
\hline & 1 & 3 & $4^{*}$ & $5^{*}$ & $9^{*}$ & 10 & $12^{*}$ & 2 & 6 & 7 & 8 & 11 & 13 & 14 \\
\hline 1 & 0.24 & 0.84 & 0.23 & 0.91 & 0.71 & 0.14 & 0.02 & 1.00 & 4.35 & 1.41 & 1.06 & 2.95 & 1.04 & 0.45 \\
\hline 2 & 0.31 & 0.80 & 0.22 & 0.54 & 0.36 & 0.07 & 0.50 & 1.24 & 2.52 & 1.03 & 0.94 & 2.42 & 0.75 & 0.36 \\
\hline 3 & 0.40 & 0.86 & 0.18 & 0.93 & 0.81 & 0.10 & 0.34 & 1.28 & 2.14 & 0.84 & 0.92 & 1.97 & 0.52 & 0.77 \\
\hline 4 & 0.39 & 1.02 & 0.16 & 1.00 & 0.86 & 1.11 & 0.25 & 1.40 & 1.60 & 0.78 & 1.12 & 1.66 & 0.39 & 0.77 \\
\hline 5 & 0.56 & 0.99 & 0.17 & 1.06 & 0.96 & 0.89 & 0.20 & 1.32 & 1.55 & 0.74 & 1.06 & 1.50 & 0.31 & 0.72 \\
\hline 6 & 0.66 & 0.98 & 0.53 & 1.04 & 0.91 & 1.36 & 0.43 & 1.23 & 1.35 & 0.75 & 0.95 & 1.41 & 0.46 & 0.77 \\
\hline 7 & 0.67 & 0.91 & 0.82 & 1.00 & 0.80 & 1.38 & 0.55 & 1.13 & 1.33 & 0.78 & 0.91 & 1.27 & 0.58 & 0.83 \\
\hline 8 & 0.76 & 0.98 & 0.98 & 1.04 & 0.86 & 1.33 & 0.62 & 1.05 & 1.28 & 0.88 & 0.88 & 1.19 & 0.61 & 0.86 \\
\hline 9 & 0.75 & 1.01 & 0.98 & 1.00 & 0.87 & 1.30 & 0.82 & 1.02 & 1.21 & 0.89 & 0.80 & 1.11 & 0.54 & 0.88 \\
\hline 10 & 0.83 & 0.98 & 0.92 & 1.01 & 1.12 & 1.19 & 0.84 & 0.98 & 1.16 & 1.03 & 0.94 & 1.09 & 0.76 & 0.91 \\
\hline 11 & 0.77 & 0.93 & 0.97 & 1.00 & 1.02 & 1.08 & 0.87 & 0.97 & 1.17 & 1.02 & 1.05 & 1.05 & 0.74 & 0.90 \\
\hline 12 & 0.93 & 0.92 & 0.94 & 1.00 & 1.02 & 0.99 & 0.94 & 1.00 & 1.13 & 1.00 & 1.16 & 1.04 & 0.76 & 0.93 \\
\hline 13 & 0.92 & 0.92 & 0.98 & 0.94 & 0.95 & 0.93 & 0.89 & & 1.08 & 1.01 & 1.08 & 1.02 & 0.98 & 0.93 \\
\hline 14 & 0.93 & 0.89 & 1.00 & 0.88 & 0.88 & 0.89 & 0.94 & & 1.04 & 1.00 & & 1.00 & 1.00 & 1.00 \\
\hline 15 & & 0.87 & & 0.82 & 0.87 & 0.83 & 1.00 & & 1.01 & & & 0.93 & & \\
\hline 16 & & & & 0.79 & 0.96 & 0.78 & & & 1.00 & & & 1.00 & & \\
\hline 17 & & & & 0.76 & 1.00 & 0.75 & & & & & & & & \\
\hline 18 & & & & 0.75 & & 0.71 & & & & & & & & \\
\hline 19 & & & & 1.00 & & 0.74 & & & & & & & & \\
\hline
\end{tabular}

${ }^{*}$ Projects that were granted an extension of time.

of permanent employees affiliated with the organization: microbusinesses should have up to ten employees; small businesses employ between eleven and fifty employees; and medium size businesses employ between 51 and 250 people. Table 3 also includes the information about the total budget and the scheduled duration for the corresponding project.

3.1. Results of Use Indexes. Table 4 includes the UI obtained for the PTM processes studied in this case. For instance, four out of the seven tasks that the Activity Definition process includes were used in Project 1 , which results a UI of 0.57 . In order to obtain UI for the schedule planning and schedule controlling functions, also shown in this table, all tasks associated with these functions were taken into account.
In Project 1 , for instance, since twenty-seven out of fiftythree schedule planning tasks were used the UI resulted 0.51, whereas eight out of twenty-four schedule controlling tasks were used the UI resulted 0.33 . Finally, the last row in this table includes the respective Total UI for every project. The calculation of the Total UI for a project took into account all PTM tasks identified in this study; for instance, since a thirtyfive out of a total of seventy-seven tasks were used, the Total UI in Project 1 resulted 0.45.

3.2. Results of Schedule Performance. Table 5 shows the weekly SPI obtained for each of the projects observed in this study. The information in Table 5 also clarifies what projects were completed on time (i.e., Projects 2, 6, 7, 8, 11, 13, and 14) as well as it indicates the projects for which an extension of 
TABLE 6: Mean SPI values obtained for different periods of time.

\begin{tabular}{|c|c|c|c|c|c|}
\hline \multirow{2}{*}{ Projects } & \multirow{2}{*}{ Performance at project completion } & \multicolumn{4}{|c|}{ Mean SPI values } \\
\hline & & 4th week & 8th week & 12th week & Last week \\
\hline 1 & Delayed & 0.33 & 0.50 & 0.61 & 0.65 \\
\hline 2 & On time & 1.00 & 1.00 & 1.00 & 1.00 \\
\hline 3 & Delayed & 0.88 & 0.92 & 0.94 & 0.93 \\
\hline 4 & Delayed* & 0.20 & 0.41 & 0.59 & 0.65 \\
\hline 5 & Delayed ${ }^{*}$ & 0.85 & 0.94 & 0.96 & 0.92 \\
\hline 6 & On time & 1.00 & 1.00 & 1.00 & 1.00 \\
\hline 7 & On time & 1.00 & 0.90 & 0.93 & 0.94 \\
\hline 8 & On time & 1.00 & 1.00 & 0.98 & 0.99 \\
\hline 9 & Delayed* & 0.68 & 0.78 & 0.86 & 0.88 \\
\hline 10 & Delayed & 0.36 & 0.80 & 0.91 & 0.87 \\
\hline 11 & On time & 1.00 & 1.00 & 1.00 & 1.00 \\
\hline 12 & Delayed* & 0.28 & 0.37 & 0.53 & 0.62 \\
\hline 13 & On time & 0.67 & 0.58 & 0.62 & 0.68 \\
\hline 14 & On time & 0.59 & 0.69 & 0.76 & 0.79 \\
\hline
\end{tabular}

${ }^{*}$ Projects that were granted an extension of time.

TABLE 7: Correlation coefficients between mean SPI values and UI values.

\begin{tabular}{lcccc}
\hline $\begin{array}{l}\text { Groups of } \\
\text { processes }\end{array}$ & 4th week & 8th week & 12th week & Last week \\
\hline $\begin{array}{l}\text { Schedule } \\
\text { planning }\end{array}$ & 0.56 & 0.41 & 0.37 & 0.40 \\
$\begin{array}{l}\text { Schedule } \\
\text { controlling }\end{array}$ & 0.56 & 0.60 & 0.58 & 0.60 \\
\begin{tabular}{l} 
Total project \\
\hline
\end{tabular} & 0.64 & 0.56 & 0.52 & 0.55 \\
\hline
\end{tabular}

time was granted to complete the project. It is important to notice that the four projects that were granted such extension (i.e., Projects 4, 5, 9, and 12) were considered as delayed.

Moreover, Table 6 includes the mean SPI values obtained by averaging the SPI values from the first week until the end of the fourth, eighth, twelfth, and last weeks (i.e., which is variable for every project, as seen in Table 5) of the project construction. For instance, to obtain the mean SPI value until the end of the fourth week of Project 1 , the SPI values (see Table 5) of the first (0.24), second (0.31), third (0.40), and fourth (0.39) weeks were averaged, resulting in a mean SPI value of 0.33 (as seen in Table 6). However, for this part of the analysis the agreement was to assume 1.0 as the maximum mean SPI value given that the main purpose was to identify those projects with satisfactory schedule performance. In addition, mean SPI values greater than 1.0 would have introduced unnecessary fluctuations into the correlation analysis in which these values were used, which would have affected the results.

The statistical relationship between the mean SPI values, obtained for the four periods of time (see Table 6), and the UI values, obtained for the planning and controlling functions as well as for the Total UI of the projects (see Table 4), was assessed by computing the respective $r$ values. Table 7 includes the correlation coefficients obtained in this case.
3.3. Results of Schedule Variance. The SV values were obtained for every week throughout the construction phase of every project. Table 8 includes the weekly SV values, makes clear what projects were completed according to the original schedule, and indicates the projects for which an extension of time was granted to complete the project. These SV results depict the number of weeks the project was ahead (i.e., the result was positive) or behind (i.e., the result was negative) of the original schedule (i.e., the Budgeted Cost of Work Scheduled).

According to the analysis with the SPI values, the mean SV values were obtained by averaging the SV values from the first week until the end of the fourth, eighth, twelfth, and last weeks of the project. Table 9 includes these results. For instance, to obtain the mean SV value until the end of the fourth week of Project 1, the SV values (see Table 8) of the first $(-0.76)$, second $(-1.38)$, third $(-1.79)$, and fourth $(-2.45)$ weeks were averaged, resulting in a mean SV value of -1.59 (as seen in Table 9).

The statistical relationship between the mean SV values, obtained for the four periods of time (see Table 9), and the UI values, obtained for the planning and controlling functions as well as for the Total UI of the projects (see Table 4), was assessed by computing the respective $r$ values. Table 10 includes the correlation coefficients obtained with this analysis.

\section{Discussion}

The results show that only $50 \%$ of the projects observed in this study were completed within the original schedule, and $21 \%$ were delayed, while for the remaining projects $(29 \%)$ an extension of time was granted to complete them. However, it is important to clarify that in all these cases such extension of time was granted given that the scope of the project was augmented; that is, changes were required by the owner to 
TABLE 8: Results of the Schedule Variance in the observed projects.

\begin{tabular}{|c|c|c|c|c|c|c|c|c|c|c|c|c|c|c|}
\hline \multirow[t]{2}{*}{ Weeks } & \multicolumn{7}{|c|}{$\begin{array}{l}\text { Schedule Variance in delayed projects } \\
\text { (results in number of weeks) }\end{array}$} & \multicolumn{7}{|c|}{$\begin{array}{l}\text { Schedule Variance in projects completed on time } \\
\text { (results in number of weeks) }\end{array}$} \\
\hline & 1 & 3 & $4^{*}$ & $5^{*}$ & $9^{*}$ & 10 & $12^{*}$ & 2 & 6 & 7 & 8 & 11 & 13 & 14 \\
\hline 1 & -0.76 & -0.16 & -0.77 & -0.09 & -0.29 & -0.86 & -0.98 & 0.00 & 3.35 & 0.41 & 0.06 & 1.95 & 0.04 & -0.55 \\
\hline 2 & -1.38 & -0.39 & -1.56 & -0.92 & -1.29 & -1.86 & -0.99 & 0.48 & 3.04 & 0.06 & -0.12 & 2.84 & -0.50 & -1.29 \\
\hline 3 & -1.79 & -0.43 & -2.47 & -0.22 & -0.57 & -2.70 & -1.99 & 0.83 & 3.42 & -0.48 & -0.24 & 2.91 & -1.44 & -0.68 \\
\hline 4 & -2.45 & 0.07 & -3.36 & 0.00 & -0.55 & 0.44 & -2.99 & 1.60 & 2.42 & -0.88 & 0.46 & 2.66 & -2.44 & -0.92 \\
\hline 5 & -2.22 & -0.03 & -4.13 & 0.32 & -0.21 & -0.56 & -3.99 & 1.59 & 2.76 & -1.32 & 0.29 & 2.52 & -3.44 & -1.40 \\
\hline 6 & -2.05 & -0.13 & -2.79 & 0.26 & -0.54 & 2.14 & -3.42 & 1.41 & 2.08 & -1.52 & -0.28 & 2.48 & -3.24 & -1.38 \\
\hline 7 & -2.29 & -0.60 & -1.26 & 0.00 & -1.38 & 2.69 & -3.14 & 0.92 & 2.32 & -1.55 & -0.63 & 1.92 & -2.91 & -1.16 \\
\hline 8 & -1.95 & -0.17 & -0.15 & 0.29 & -1.13 & 2.65 & -3.00 & 0.36 & 2.26 & -0.93 & -0.99 & 1.52 & -3.10 & -1.12 \\
\hline 9 & -2.25 & 0.09 & -0.21 & 0.01 & -1.16 & 2.66 & -1.62 & 0.21 & 1.89 & -0.96 & -1.81 & 1.01 & -4.10 & -1.10 \\
\hline 10 & -1.73 & -0.22 & -0.75 & 0.09 & 1.17 & 1.90 & -1.57 & -0.21 & 1.64 & 0.30 & -0.58 & 0.89 & -2.38 & -0.90 \\
\hline 11 & -2.48 & -0.74 & -0.30 & 0.00 & 0.27 & 0.90 & -1.38 & -0.31 & 1.88 & 0.17 & 0.60 & 0.52 & -2.83 & -1.10 \\
\hline 12 & -0.81 & -0.99 & -0.68 & 0.00 & 0.22 & -0.10 & -0.71 & 0.00 & 1.52 & 0.01 & 1.89 & 0.48 & -2.82 & -0.84 \\
\hline 13 & -1.00 & -1.06 & -0.28 & -0.74 & -0.70 & -0.97 & -1.47 & & 1.09 & 0.10 & 1.00 & 0.31 & -0.28 & -0.92 \\
\hline 14 & -1.00 & -1.53 & 0.00 & -1.66 & -1.70 & -1.50 & -0.78 & & 0.56 & 0.00 & & 0.00 & 0.00 & 0.00 \\
\hline 15 & & -2.00 & & -2.65 & -1.91 & -2.49 & 0.00 & & 0.11 & & & -1.00 & & \\
\hline 16 & & & & -3.37 & -0.58 & -3.48 & & & 0.00 & & & 0.00 & & \\
\hline 17 & & & & -4.10 & 0.00 & -4.27 & & & & & & & & \\
\hline 18 & & & & -4.48 & & -5.22 & & & & & & & & \\
\hline 19 & & & & 0.00 & & -5.00 & & & & & & & & \\
\hline
\end{tabular}

* Projects that were granted an extension of time.

TABLE 9: Mean SV values obtained for different periods of time (variance in number of weeks).

\begin{tabular}{|c|c|c|c|c|c|}
\hline \multirow{2}{*}{ Projects } & \multirow{2}{*}{ Performance at project completion } & \multicolumn{4}{|c|}{ Mean SPI values } \\
\hline & & 4th week & 8th week & 12th week & Last week \\
\hline 1 & Delayed & -1.59 & -1.86 & -1.85 & -1.73 \\
\hline 2 & On time & 0.73 & 0.90 & 0.57 & 0.57 \\
\hline 3 & Delayed & -0.23 & -0.23 & -0.31 & -0.55 \\
\hline 4 & Delayed* & -2.04 & -2.06 & -1.53 & -1.34 \\
\hline 5 & Delayed* & -0.31 & -0.04 & -0.02 & -0.90 \\
\hline 6 & On time & 3.06 & 2.71 & 2.38 & 1.90 \\
\hline 7 & On time & -0.22 & -0.78 & -0.56 & -0.47 \\
\hline 8 & On time & 0.04 & -0.18 & -0.11 & -0.03 \\
\hline 9 & Delayed* & -0.67 & -0.74 & -0.45 & -0.61 \\
\hline 10 & Delayed & -1.24 & 0.25 & 0.61 & -0.82 \\
\hline 11 & On time & 2.59 & 2.35 & 1.81 & 1.31 \\
\hline 12 & Delayed ${ }^{*}$ & -1.74 & -2.56 & -2.15 & -1.87 \\
\hline 13 & On time & -1.09 & -2.13 & -2.43 & -2.10 \\
\hline 14 & On time & -0.86 & -1.06 & -1.04 & -0.96 \\
\hline
\end{tabular}

${ }^{*}$ Projects that were granted an extension of time.

include deliverables that were not considered in the original project. This may be associated with a common situation observed in the context studied in this case: projects passing to the bidding phase without having the planning and design phases properly completed.

Although the sample of projects analyzed in this case was small and the number of firms in each of the size groups (micro, small, and medium) was uneven, it was possible to observe that projects completed within the original schedule were mostly (six of seven projects) in charge of small and medium contractors, while on the other hand only one of the projects executed by a microbusiness was concluded on time. This outcome is deemed reasonable considering the usual lack of an organizational structure in microbusinesses, 
TABLE 10: Correlation coefficients between mean SV values and UI values.

\begin{tabular}{lcccc}
\hline \multirow{2}{*}{ Groups of processes } & \multicolumn{4}{c}{ Periods of time } \\
& 4th week & 8th week & 12th week & Last week \\
\hline Schedule planning & 0.41 & 0.30 & 0.27 & 0.37 \\
Schedule controlling & 0.46 & 0.49 & 0.47 & 0.51 \\
Total project & 0.50 & 0.43 & 0.40 & 0.49 \\
\hline
\end{tabular}

which makes it difficult to attain the performance that can be observed in larger contractors.

The Total UI of the projects were distributed around a mean value of 0.47 and a range from 0.34 to 0.65 , which indicates that, in average, only half of tasks recommended for planning and controlling project time were used in this case. Five out of the six projects, with a Total UI equal or greater than such mean value, were completed within the original schedule (i.e., Projects 2, 6, 7, 8, and 11, as seen in Table 4). However, two of the projects with a Total UI smaller than the mean value of 0.51 were completed within the original schedule as well (i.e., Projects 13 and 14). Moreover, Figures 2 and 3 depict the distributions of the SV values (see Table 8), respectively, obtained for the group of projects with Total UI values greater than the mean Total UI (i.e., 0.51) and for the group of projects with Total UI values smaller than the mean Total UI. The former group of projects resulted with a mean SV value of 0.38 (i.e., projects completed on time as indicated by the positive mean SV value), while the last one resulted with a mean SV value of -1.21 (i.e., projects delayed as indicated by the negative mean SV value). Notice, by comparing both histograms, that projects with Total UI values greater than the mean Total UI (see Figure 2) have SV values biased towards the positive range of SV values.

The statistical dependence between the UI values and the schedule performance values (i.e., SPI and SV values) was significant though mostly moderate, as seen in Tables 7 and 10. Actually, a previous study conducted by Yean and Theng [14] proved a significant correlation between sixteen practices associated with controlling and the performance of the projects. They identified that the most relevant controlling practices include appropriate use of project information for developing the project schedule, proper use of activity floats for scheduling, and the quality of techniques supporting identification of project risks. Yean and Theng also emphasized that good practices for project management and adequate resources are not enough for attaining good performance in projects, but on-site control systems are also required to accomplish performance objectives of construction projects. On the other hand, results in this study evidenced a significant statistical relationship between the use of practices recommended for project planning and controlling and project performance.

\section{Conclusions}

The results of study reported in this paper indicate a significant effect of the use of processes related to Project Time Management on project performance, especially for

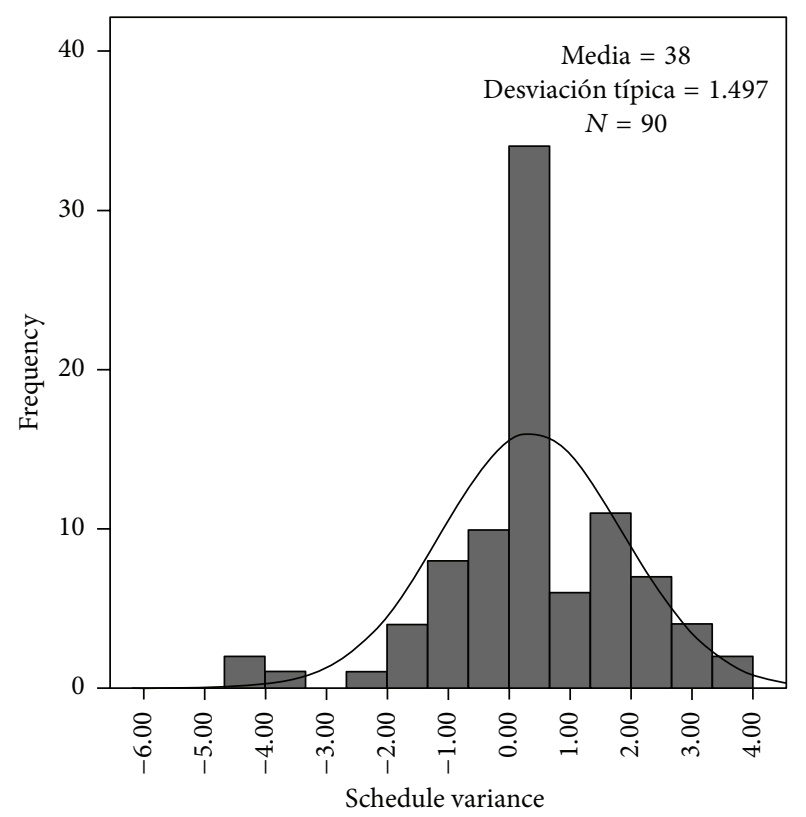

FIGURE 2: Frequencies of SV values in projects with UI values greater than the mean value of UI.

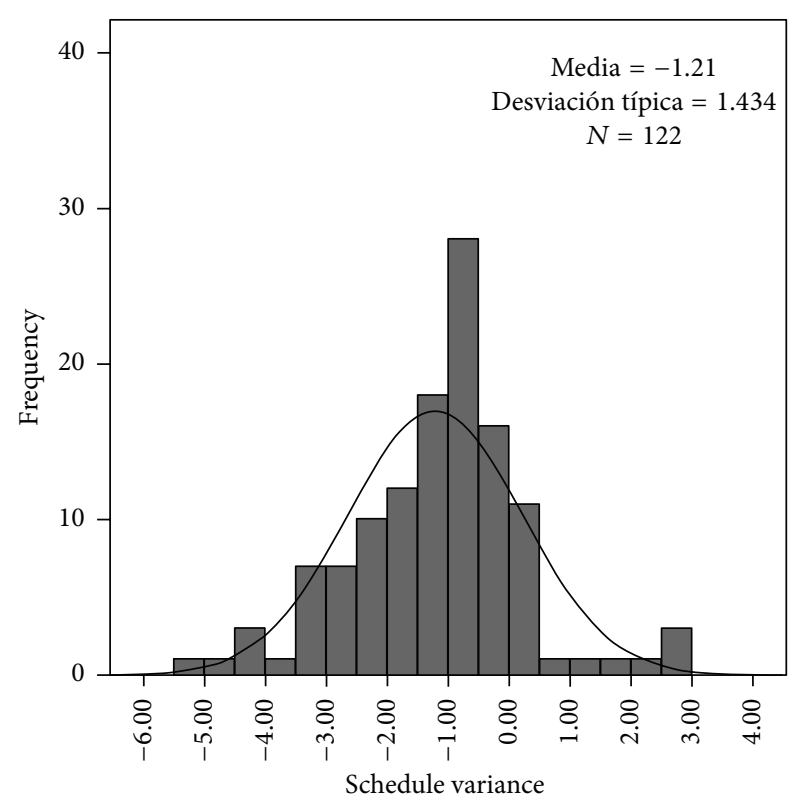

FIGURE 3: Frequencies of SV values in projects with UI values smaller than the mean value of UI.

completing the construction phase within the original schedule. However, the results obtained from the assessment of both variables demonstrate a mediocre level of project management.

The use of PTM processes was assessed with a Use Index obtained with the responses provided by managers of fourteen projects, who selected from a list of seventy-seven tasks associated with schedule planning and controlling those that had been used in their own projects. In this case, the mean value obtained for the Use Index of these projects 
was 0.47 , which evidenced a poor application of project management, or at least of Project Time Management. This result is consistent with other previous studies that found that poor project planning and controlling are one of the main causes of project delays.

On the other hand, project schedule performance was assessed with the Schedule Performance Index and the Schedule Variance obtained with the Earned Schedule method. According to the SPI and SV measures obtained in this study, only seven out of fourteen projects were completed within the original schedule. The other seven projects were delayed though four of them were granted an extension of time to complete the construction works. This extension was actually attributable to the owner since an augmentation of the project scope was required. This also coincides with other studies in which the changes required by owners were found to be a major cause of project delays.

However, despite poor project management observed in these projects, it was proved that the more effort dedicated to project schedule management the more likely to succeed on timely completion of construction projects. In most of the projects in which completion was attained on time, a greater amount of tasks associated with schedule planning and controlling was used.

\section{Conflict of Interests}

The authors declare that there is no conflict of interests regarding the publication of this paper.

\section{References}

[1] A. Al-Kharashi and M. Skitmore, "Causes of delays in Saudi Arabian public sector construction projects," Construction Management and Economics, vol. 27, no. 1, pp. 3-23, 2009.

[2] J. González, R. Solís, and C. Alcudia, "Diagnóstico sobre la Planeación y Control de Proyectos en las PYMES de Construcción," Revista de la Construcción, vol. 9, no. 1, pp. 17-25, 2010.

[3] M. Marzouk, A. El-Dokhmasey, and M. El-Said, "Assessing construction engineering-related delays: Egyptian perspective," Journal of Professional Issues in Engineering Education and Practice, vol. 134, no. 3, pp. 315-326, 2008.

[4] R. Singh, "Cost and time overruns in infrastructure projects: extent, causes and remedies," Working Paper 181, Department of Economics, University of Delhi, Nueva Deli, India, 2009.

[5] J. Woodward, Construction Project Management: Getting it Right First Time, Thomas Telford, London, UK, 1997.

[6] CIOB, Code of Practice for Project Management for Construction and Development, Chartered Institute of Building, Blackwell, Oxford, UK, 3rd edition, 2002.

[7] Project Management Institute, A Guide to the Project Management Body of Knowledge (PMBOK Guide), Project Management Institute, Philadelphia, Pa, USA, 5th edition, 2013.

[8] Association for Project Management, APM Body of Knowledge, Association for Project Management, Buckinghamshire, UK, 6th edition, 2012.

[9] I. Liviu, E. Crisan, and I. Muresan, "Best practices in project management," Review of International Comparative Management, vol. 11, no. 1, pp. 43-51, 2010.
[10] R. Solís, G. Martínez, and J. González, "Estudio de Caso: Demoras en la construcción de un Proyecto en México," Ingeniería, Revista Académica de la FIUADY, vol. 13, no. 1, pp. 41-48, 2009.

[11] W. Lipke, "Schedule is different," The Measurable News, pp. 7-9, 2003.

[12] H. Wadsworth, Handbook of Statistical Methods for Engineers and Scientists, McGraw-Hill, 2nd edition, 1998.

[13] INEGI, Micro, Small, Medium, and Large Businesses: Stratification according to 2009 Economic Census, National Institute for Statistics and Geography, Aguascalientes, Mexico, 2009.

[14] F. Yean and W. Theng, "Using control systems to improve construction project outcomes," Engineering, Construction and Architectural Management, vol. 20, no. 6, Article ID 17098758, pp. 576-588, 2013. 

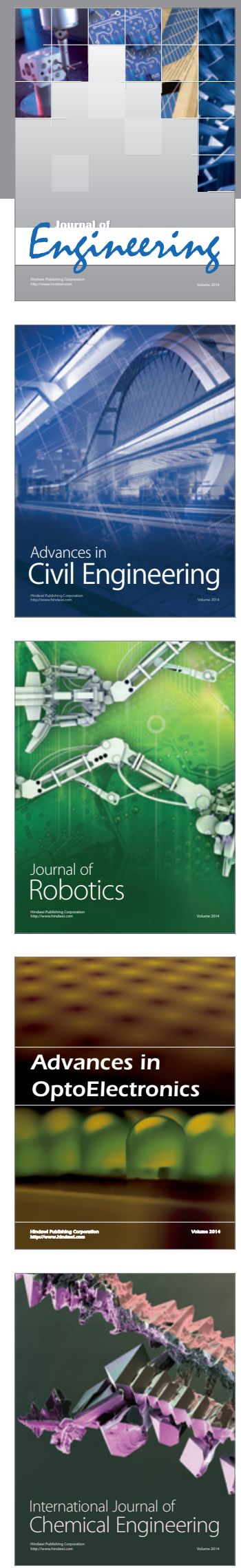

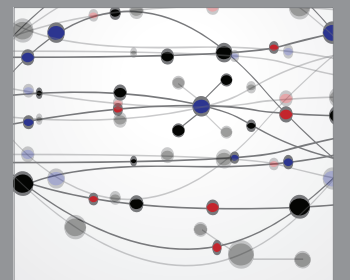

The Scientific World Journal
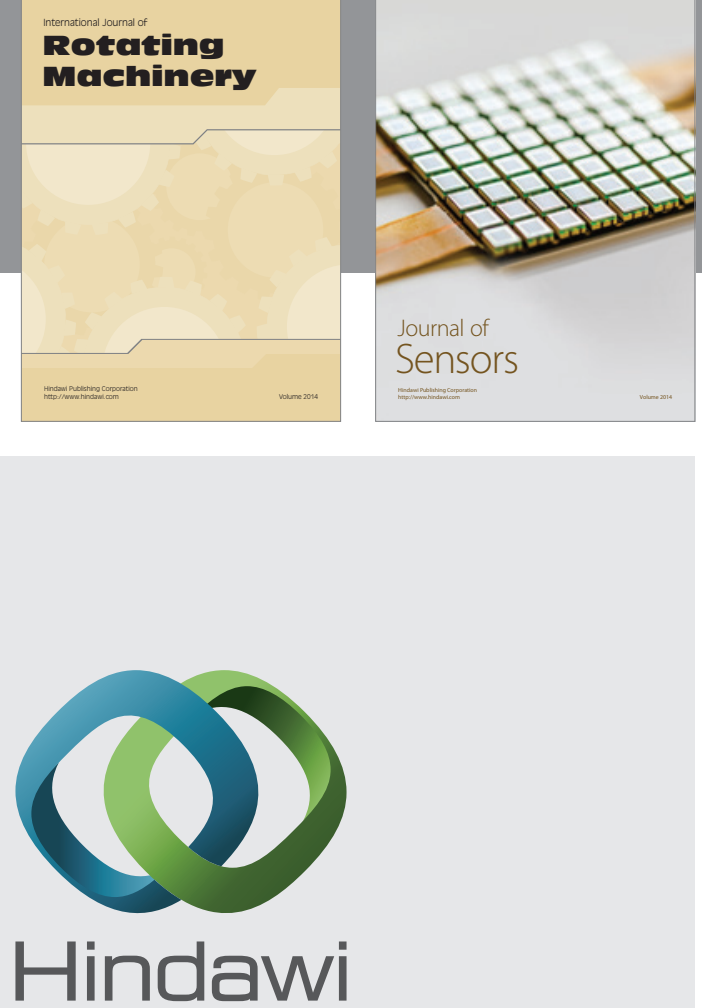

Submit your manuscripts at http://www.hindawi.com
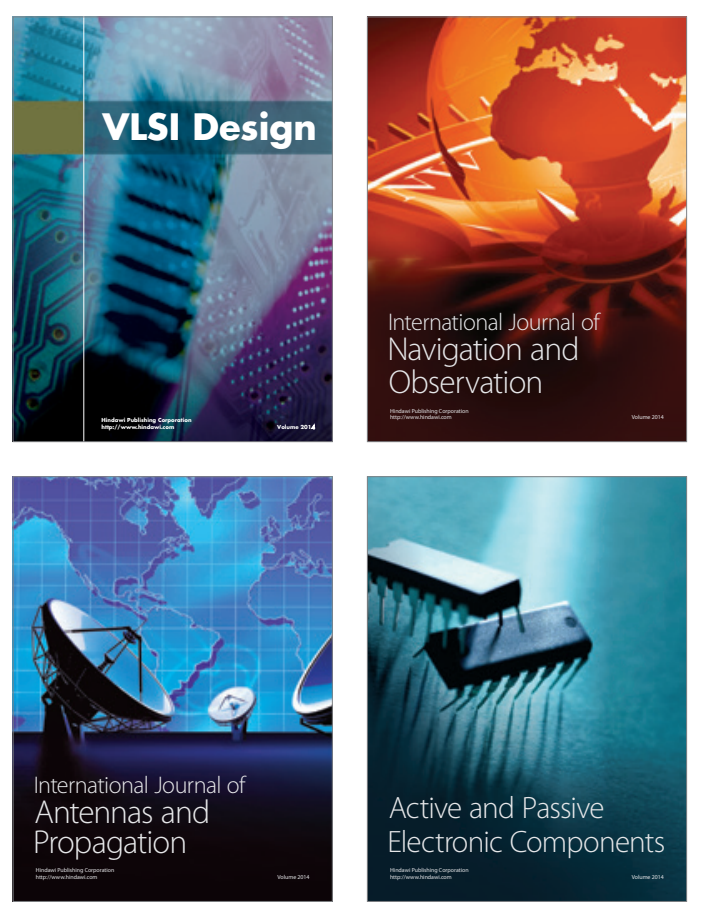
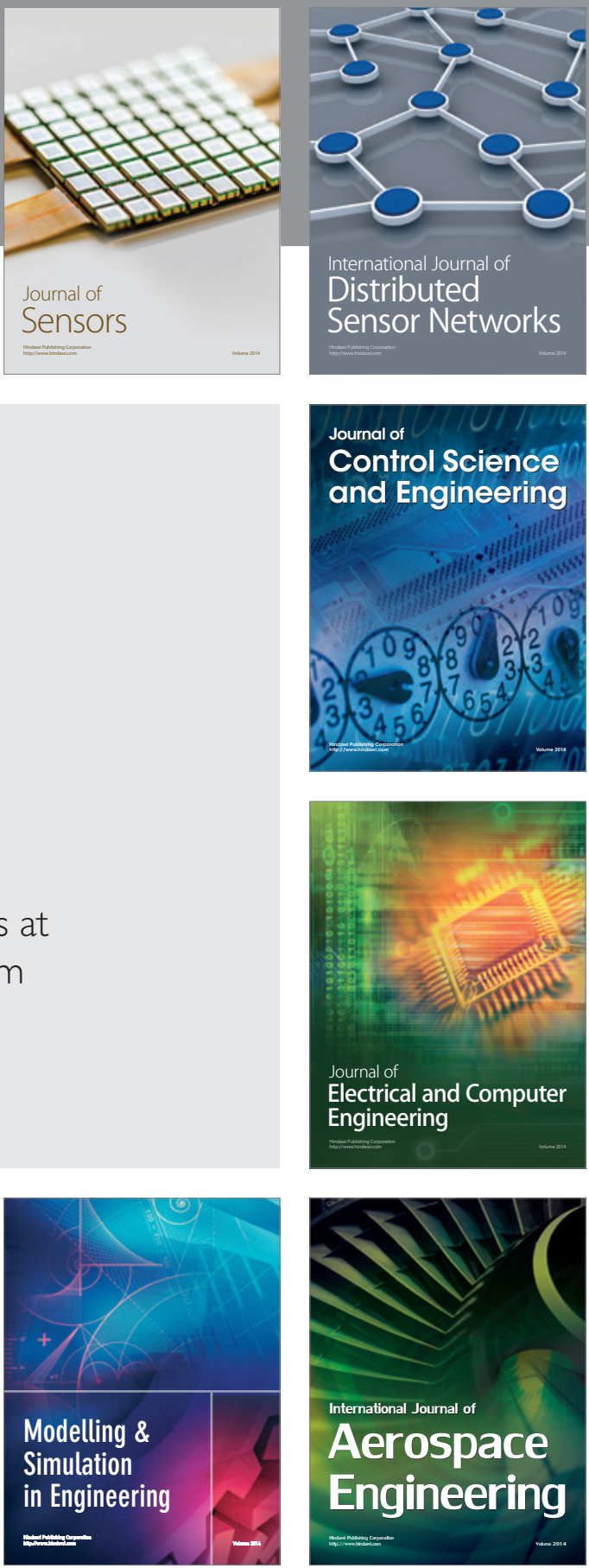

Journal of

Control Science

and Engineering
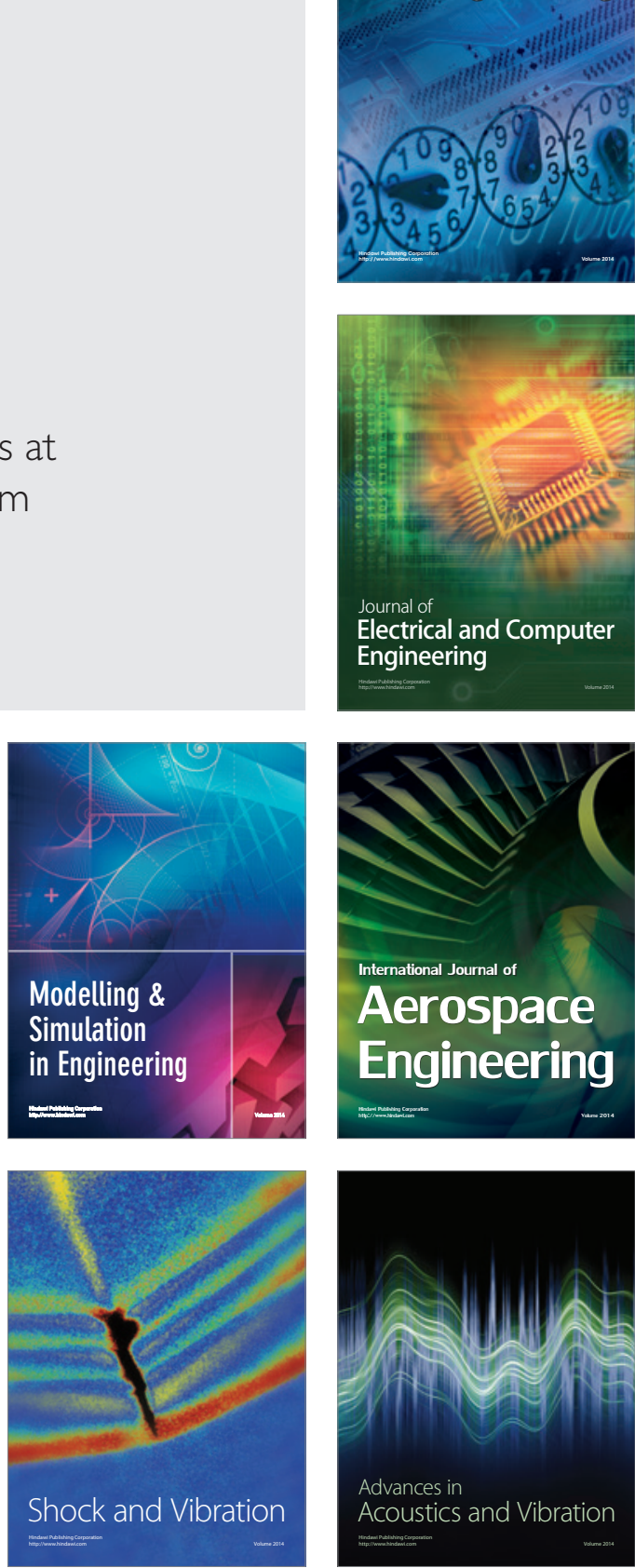